\title{
Supramolecular Assemblies and Redox Modulation of Pyromellitic Diimide-Based Cyclophane via Noncovalent Interactions with Naphtol
}

\author{
Shin-ichiro Kato, ${ }^{\dagger, \downarrow}$ Taisuke Matsumoto ${ }^{\S}$ Keiko Ideta,${ }^{\S}$ Toshiaki Shimasaki, ${ }^{\dagger, \downarrow}$ Kenta Goto, ${ }^{\dagger}$ \\ Teruo Shinmyozu $*^{\dagger}$ \\ ${ }^{\dagger}$ Institute for Materials Chemistry and Engineering (IMCE) and ${ }^{\ddagger}$ Department of Molecular Chemistry, \\ Graduate School of Sciences, Kyushu University, 6-10-1 Higashi-ku, Fukuoka 812-8581, ${ }^{\S}$ Institutefor Materials \\ Chemistry and Engineering (IMCE), 6-1 Kasuga-koh-en, Kasuga 816-8580, Japan
}

\section{Contents}

1. General experimental methods (S2)

2. Dynamic behaviour of $\mathbf{1}$ in $\mathrm{CD}_{2} \mathrm{Cl}_{2}$ solution

Figure S1. Temperature-dependent ${ }^{1} \mathrm{H} N \mathrm{NR}$ spectra of $\mathbf{1}$ in $\mathrm{CD}_{2} \mathrm{Cl}_{2}(\mathrm{~S} 3)$

3. Complexation studies

Figure S2. UV/Vis spectra of $\mathbf{1}$ and $\mathbf{1}$ plus $\beta$-naphtol in chloroform (S4)

Figure S3. UV/Vis spectra of $\mathbf{1}$ and $\mathbf{1}$ plus indole in chloroform (S4)

Figure S4. UV/Vis spectra of $\mathbf{1}$ and $\mathbf{1}$ plus $p$-dimethoxybenzene in chloroform (S5)

Figure S5. UV/Vis spectra of $\mathbf{1}$ and $\mathbf{1}$ plus $p$-xylene in chloroform (S5)

Figure S6. $\quad{ }^{1} \mathrm{H}$ NMR observation of $\mathbf{1}$ recorded in $\mathrm{CDCl}_{3}$ in the presence of (a) $\beta$-naphtol and (b) indole (S6)

Figure S7. IR spectra of 1 plus $\beta$-naphtol in chloroform (S6-S7)

Figure S8 ${ }^{1} \mathrm{H}$ NMR spectra (300 MHz) of $\mathbf{1} / \alpha$-naphtol $/ \beta$-naphtol complex obtained from competitive co-crystallization experiments (S7) 
4. X-ray Crystallographic Structure Analyses (S8)

Figure S9. ORTEP drawings for 1 and $\alpha$-naphtol (S9)

Figure S10. ORTEP drawings for 1 and $\beta$-naphtol (S10)

5. Electrochemistry

Figure S11. $\quad C V$ traces for 1 in the presence of $\beta$-naphtol (S10)

Figure S12. $\quad \mathrm{CV}$ traces for $\mathbf{2}$ in the presence of $\alpha$-naphtol (S11)

Figure S13. $\quad \mathrm{CV}$ traces for $\mathbf{2}$ in the presence of $\beta$-naphtol (S11)

6. Theoretical methods

Table S1. Coordinates of syn-1' (S11)

Table S2. Coordinates of anti-1' (S13)

Table S3. Coordinates of parallel-1' (S15)

Table S4. Coordinates of 1 (S17)

Table S5. Coordinates of 2 (S19)

7. References (S20) 


\section{General experimental methods}

IR spectra were measured as $\mathrm{KBr}$ pellets. ${ }^{1} \mathrm{H} \mathrm{NMR}$ spectra were determined in $\mathrm{CDCl}_{3}, \mathrm{DMSO}-d_{6}$, or $\mathrm{CD}_{2} \mathrm{Cl}_{2}$. Chemical shifts $(\delta)$ are given as $\delta$ values (ppm) relative to tetramethylsilane (TMS). The coupling constants $(J)$ are given in hertz. FAB-MS spectra were recorded with $m$-nitrobenzyl alcohol as a matrix. UV/Vis spectra were measured in a $1 \mathrm{~cm}$ width quartz cell. Cyclic voltanmmetry was performed by using a cell equipped with a glassy carbon as working electrode, a platinum wire as counter electrodes, and $\mathrm{Ag} / \mathrm{AgNO}_{3}$ as the referential electrode. All electrochemical measurements were performed in dichloromethane solution $\left(5 \times 10^{-4}\right.$ mmol dm${ }^{-3}$ ) containing $0.10 \mathrm{M}$ tetra- $n$-butylammonium hexafluorophosphate at room temperature. THF was distilled from sodium and benzophenone under an argon atmosphere just before use. NMP and acetone were dried with 4A molecular sieve over night before use. Resorcinol 3, pyromellitic dianhydride 8, and 2-methoxybenzylamine $\mathbf{9}$ are commercially available. 1,3-Di- $n$-butoxybenzene $\quad \mathbf{4}^{1}$ 1,3-di- $n$-butoxy-4,6-diiodobenzene $5,{ }^{2}$ and 1,3-di- $n$-butoxy-4,6-dicyanobenzene $\mathbf{6}^{2}$ were prepared according to the method described previously. $\alpha$-Naphtol and $\beta$-naphtol were recrystallized from $\mathrm{CHCl}_{3}$ and hexane before use.

\section{Dynamic behaviour of 1 in $\mathrm{CD}_{2} \mathrm{Cl}_{2}$ solution}

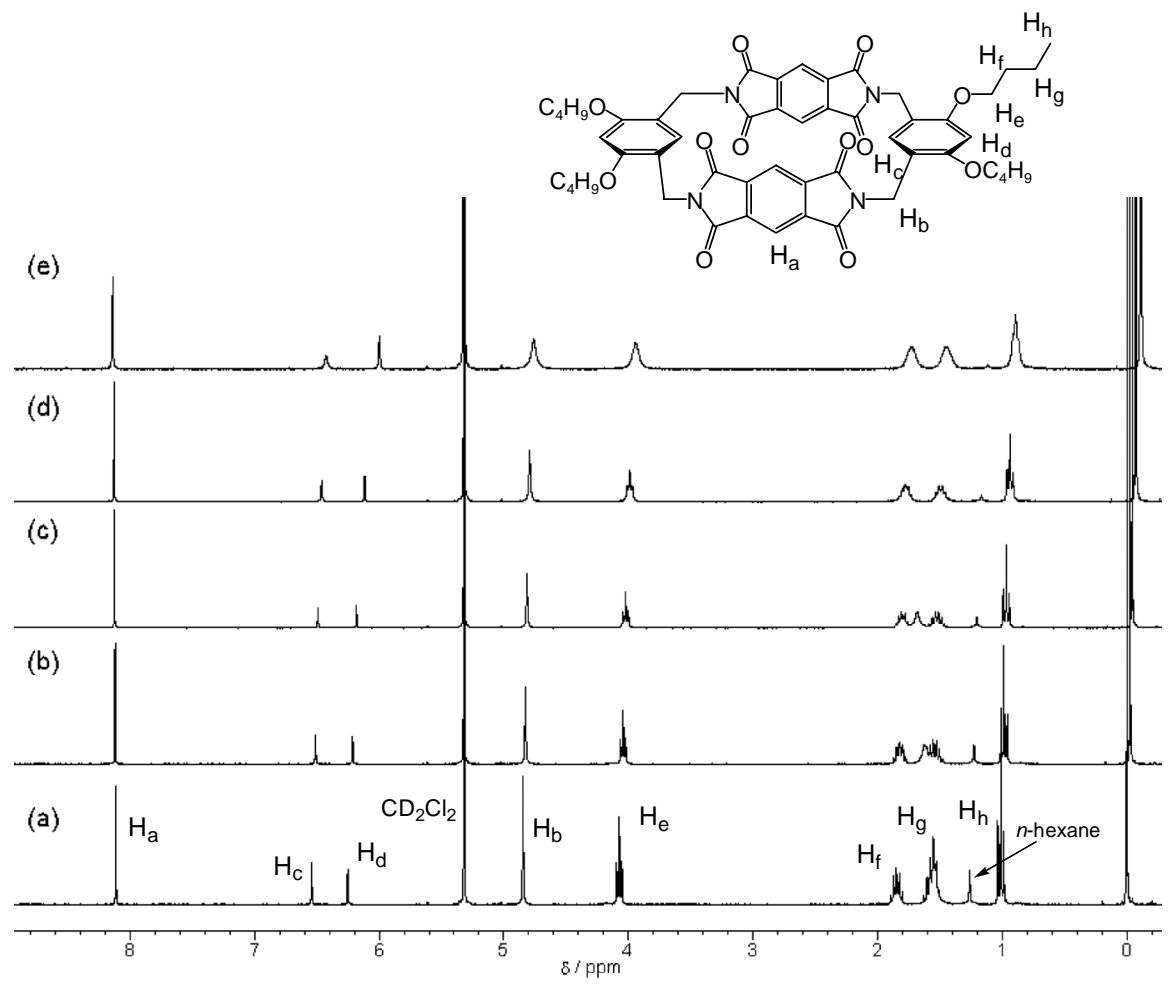

Figure S1. Temperature-dependent ${ }^{1} \mathrm{H}$ NMR spectra of 1 in $\mathrm{CD}_{2} \mathrm{Cl}_{2}$ : (a) $25^{\circ} \mathrm{C},(\mathrm{b})-10{ }^{\circ} \mathrm{C}$, (c) $-30{ }^{\circ} \mathrm{C}$, (d) -60 ${ }^{\circ} \mathrm{C},(\mathrm{e})-100{ }^{\circ} \mathrm{C}$. 


\section{Complexation studies}
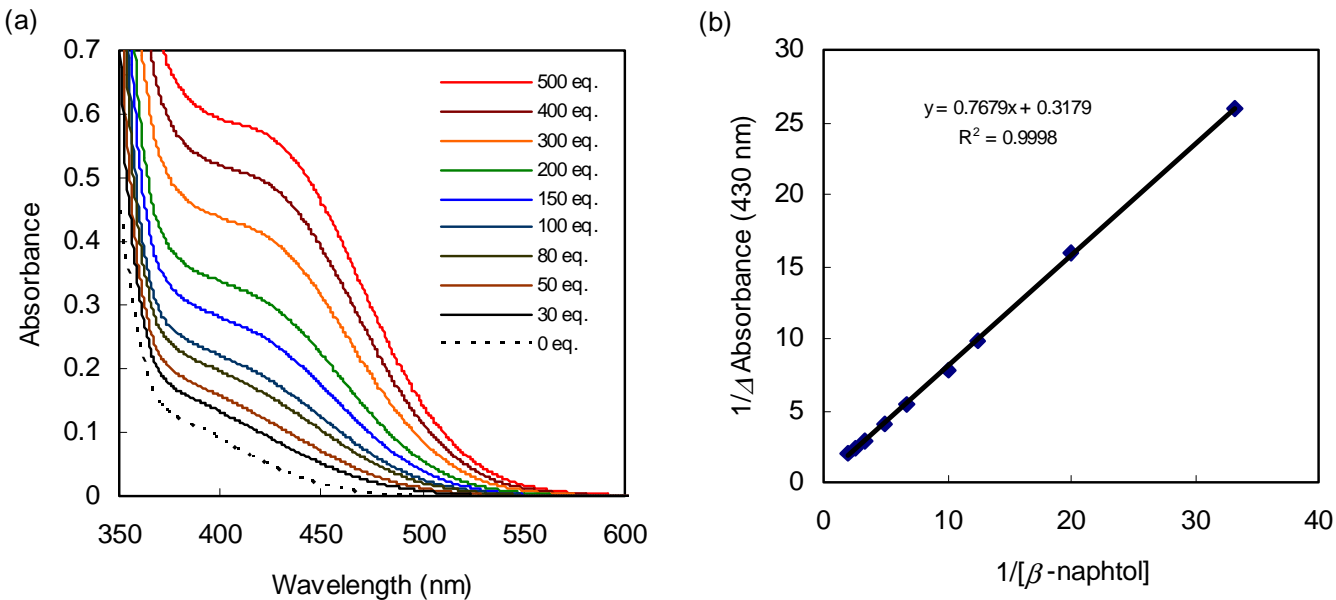

Figure S2. (a) Absorption spectra of 1 in $\mathrm{CHCl}_{3}$ at $1 \times 10^{-3} \mathrm{M}$ in the presence of $0-500$ equiv of $\beta$-naphtol. (b) Benesi-Hildebrand plot.
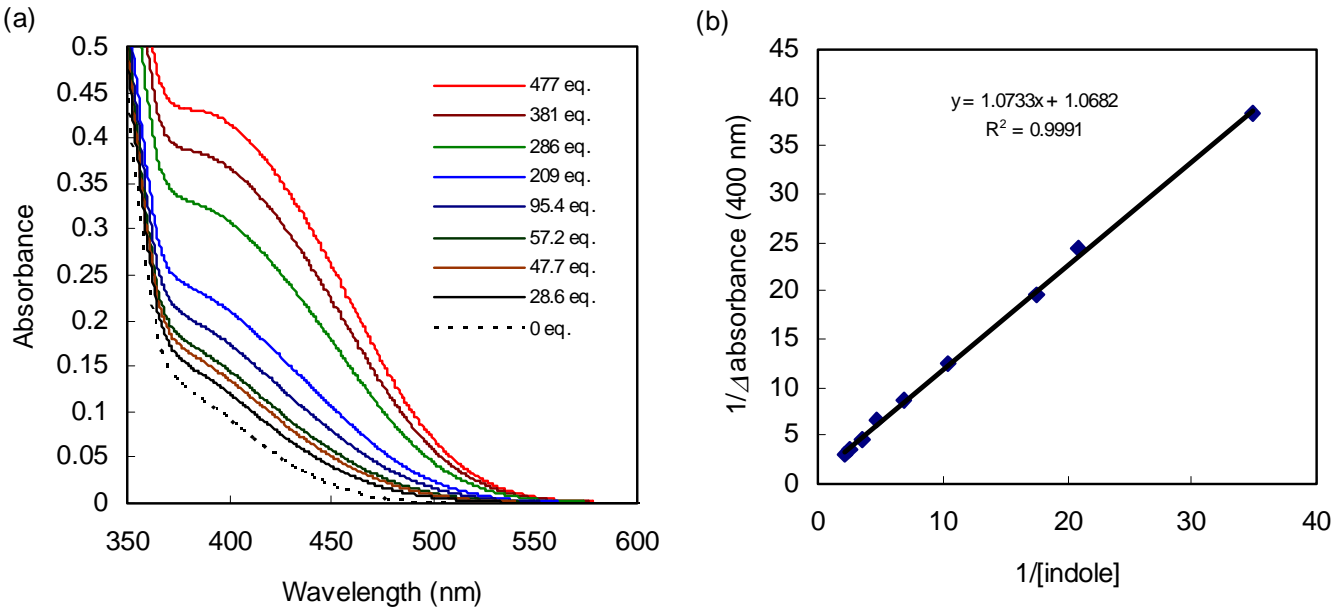

Figure S3. (a) Absorption spectra of 1 in $\mathrm{CHCl}_{3}$ at $1 \times 10^{-3} \mathrm{M}$ in the presence of $0-477$ equiv of indole. (b) Benesi-Hildebrand plot. 

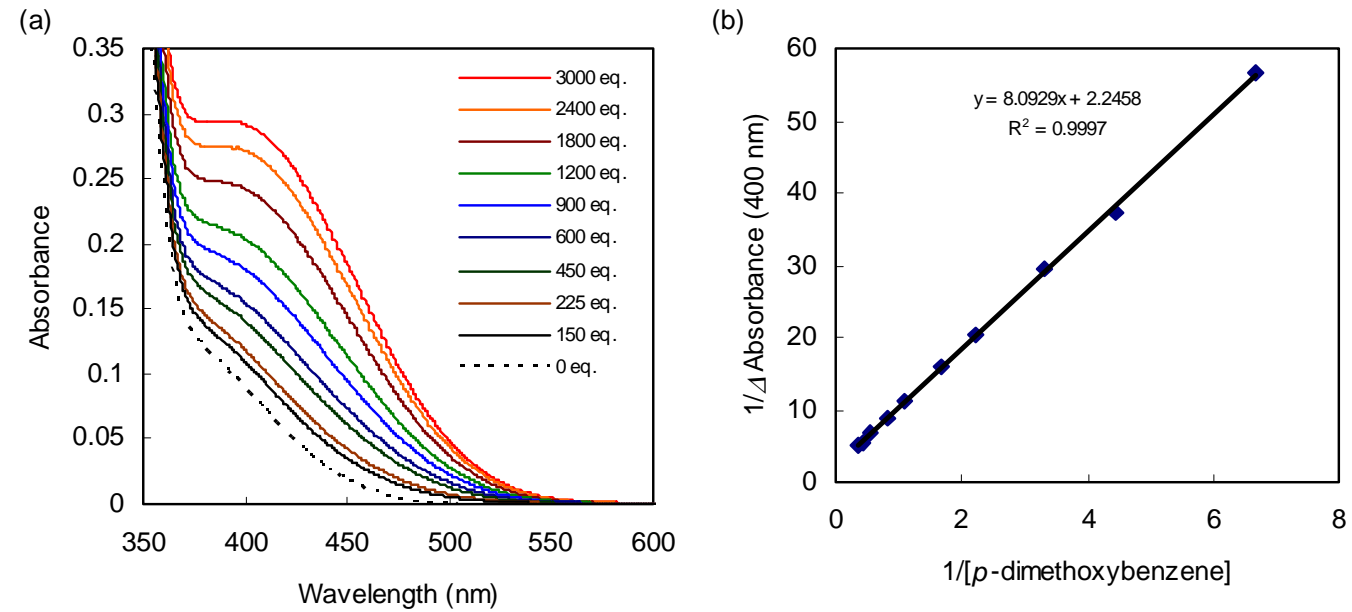

Figure S4. (a) Absorption spectra of 1 in $\mathrm{CHCl}_{3}$ at $1 \times 10^{-3} \mathrm{M}$ in the presence of $0-3000$ equiv of p-dimethoxybenzene. (b) Benesi-Hildebrand plot.

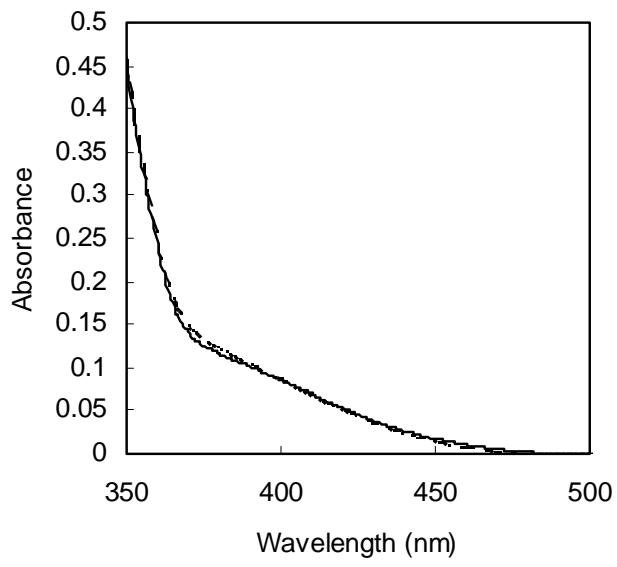

Figure S5. UV/Vis spectra of $\mathbf{1}$ (solid line) and $\mathbf{1}$ plus $p$-xylene (dashed line) in $\mathrm{CHCl}_{3} . \quad[\mathbf{1}]=1 \times 10^{-3} \mathrm{M}$, $[p$-xylene $]=0.1 \mathrm{M}$. 

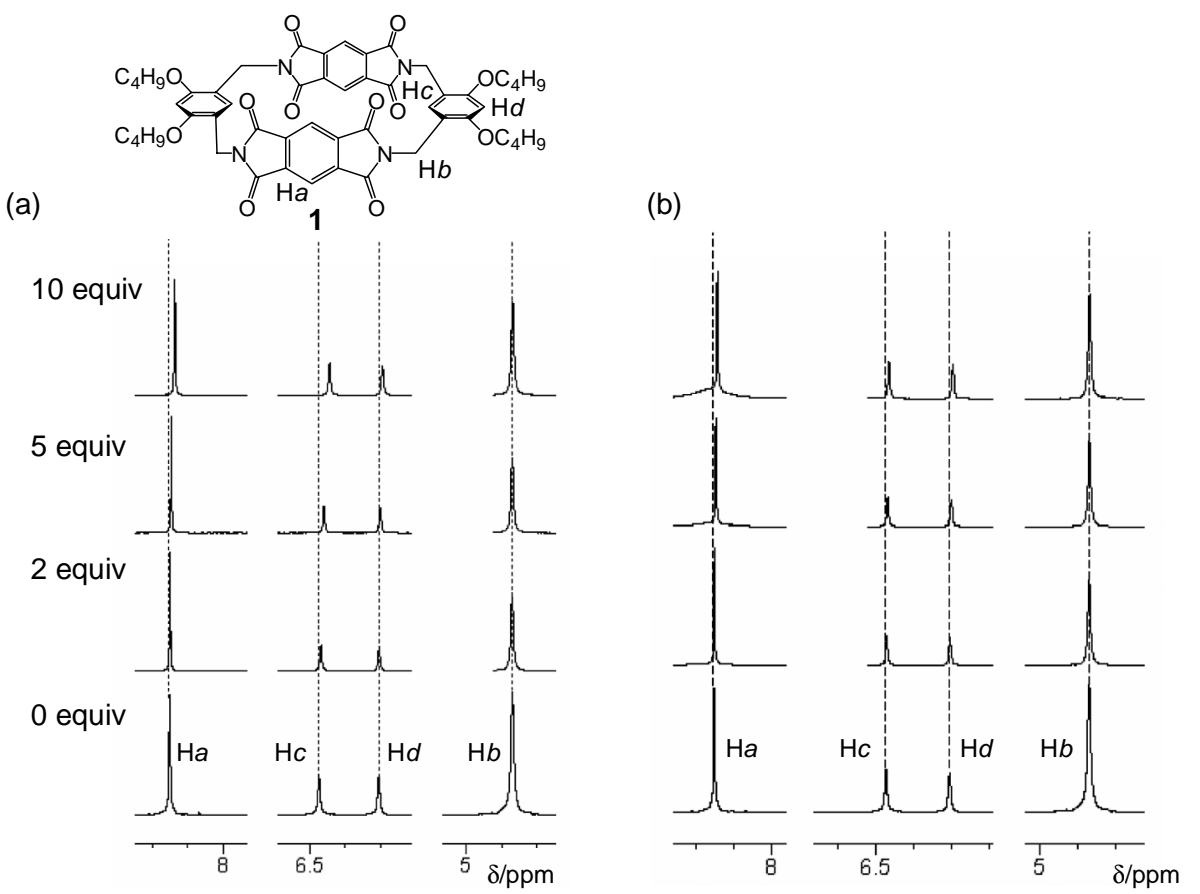

Figure S6. ${ }^{1} \mathrm{H}$ NMR observation of 1 recorded in $\mathrm{CDCl}_{3}$ ( $300 \mathrm{MHz}, 25{ }^{\circ} \mathrm{C}$, TMS as an external standard) in the presence of (a) $\beta$-naphtol and (b) indole.

(a)

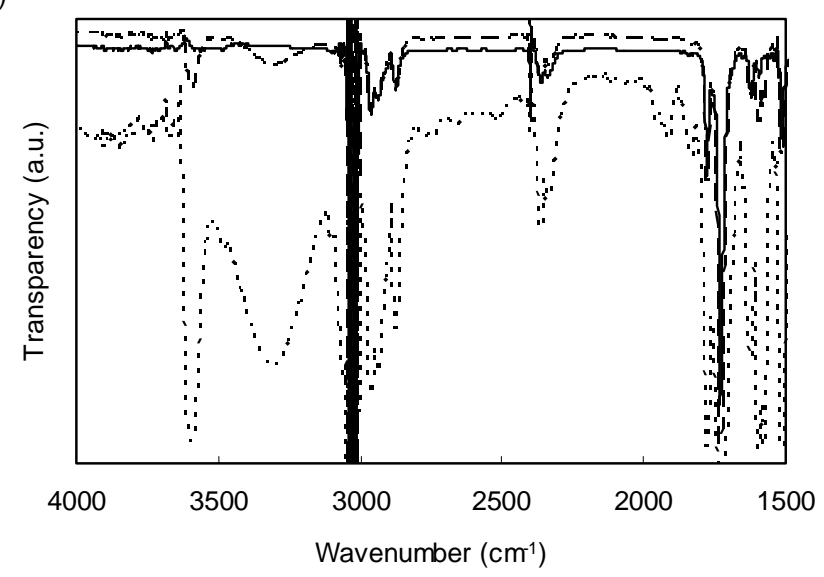




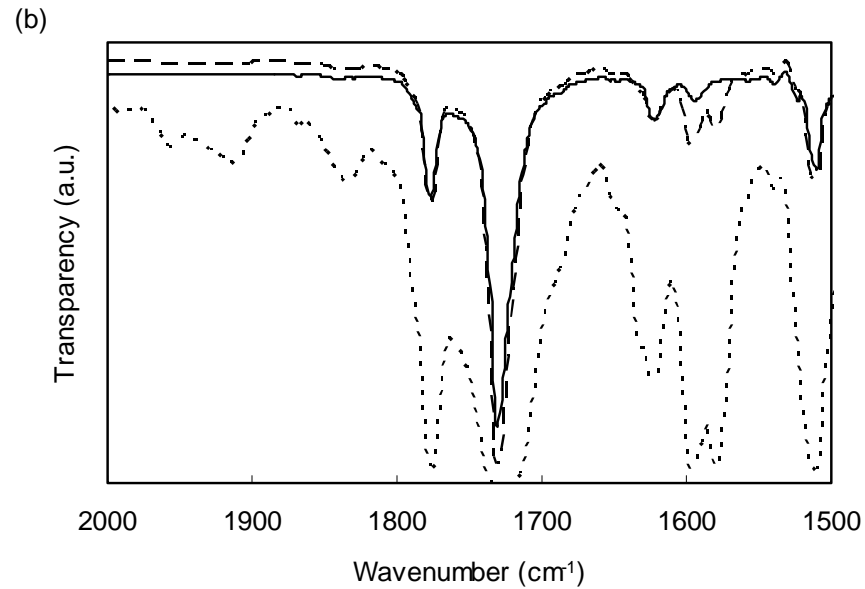

Figure S7. (a) IR spectra of $\mathbf{1}$ (solid line: [1] $=1 \times 10^{-3} \mathrm{M}$ ), $\mathbf{1}$ plus $\beta$-naphtol (dashed line: $[\mathbf{1}]=1 \times 10^{-3} \mathrm{M}$, $[\beta$-naphtol $]=1 \times 10^{-2} \mathrm{M}$ ), and $\mathbf{1}$ plus $\beta$-naphtol (dotted line: $[\mathbf{1}]=1 \times 10^{-2} \mathrm{M},[\beta$-naphtol $\left.]=0.1 \mathrm{M}\right)$ in $\mathrm{CHCl}_{3}$. (b) Magnified figure around $1500-2000 \mathrm{~cm}^{-1}$.
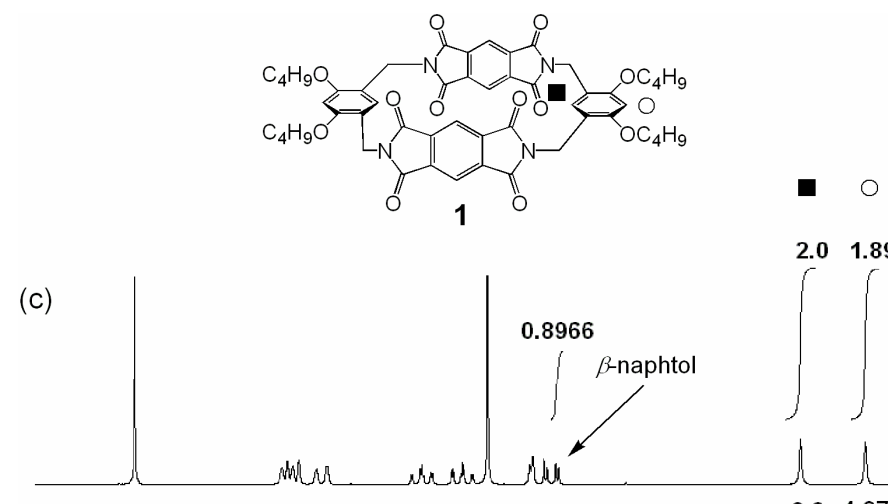

(b)
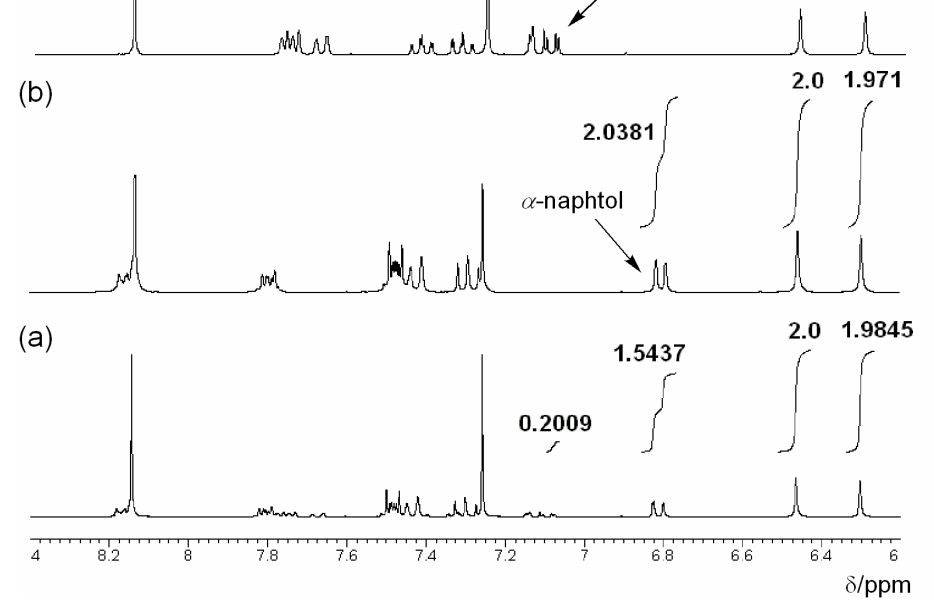

Figure S8. (a) ${ }^{1} \mathrm{H}$ NMR spectra (300 MHz) in $\mathrm{CDCl}_{3}$ of $\mathbf{1} / \alpha$-naphtol $/ \beta$-naphtol complexes obtained from competitive co-crystallization experiment. (b) ${ }^{1} \mathrm{H} \mathrm{NMR}$ spectra (300 MHz) in $\mathrm{CDCl}_{3}$ of $\mathbf{1}\left(1 \times 10^{-2} \mathrm{M}\right)$ in the presence of $\alpha$-naphtol $\left(2 \times 10^{-2} \mathrm{M}\right)$. (c) ${ }^{1} \mathrm{H} \mathrm{NMR}(300 \mathrm{MHz})$ spectra in $\mathrm{CDCl}_{3}$ of $\mathbf{1}\left(1 \times 10^{-2} \mathrm{M}\right)$ in the presence of $\beta$-naphtol $\left(2 \times 10^{-2} \mathrm{M}\right) . \quad \mathbf{\square}=$ inner benzene protons. $\bigcirc=$ outer benzene protons. 


\section{X-ray Crystallographic Structure Analyses}

X-ray crystallography was performed on a Rigaku RAXIS RAPID imaging plate diffractometer with graphite monochromated Mo K $\alpha$ radiation $(\lambda=0.71070 \AA)$ for $1 \cdot(\mathrm{DMF})_{2}$ and on a Rigaku Saturn CCD area detector with graphite monochromated Mo K $\alpha$ radiation $(\lambda=0.71070 \AA)$ for $\mathbf{1} \cdot(\alpha \text {-naphtol })_{2}$ and $\mathbf{1} \cdot \beta$-naphtol. The data were collected at $113 \mathrm{~K}$ using $\omega$ scan in the $\theta$ range of 2.2-27.6 deg for $1 \cdot(\mathrm{DMF})_{2}$ and at $123 \mathrm{~K}$ using $\omega$ scan in the $\theta$ range of 1.8-27.6 deg for $\mathbf{1} \cdot(\alpha \text {-naphtol })_{2}$ and $\mathbf{1} \cdot \beta$-naphtol. The structures were solved by direct method (SIR97) and were refined using full-matrix least squares (teXsan for 1-(DMF) $)_{2}$ and CRYSTALS for 1. $(\alpha \text {-naphtol })_{2}$ and 1. $\beta$-naphtol) based on $F^{2}$ of all independent reflections measured. All $\mathrm{H}$ atoms were located at ideal positions and refined as riding on their parent atoms with $U_{\text {iso }}(\mathrm{H})=1.2-1.5 U_{\text {eq }}(\mathrm{C})$ and with $\mathrm{C}-\mathrm{H}=0.95$ A. Crystallographic data for the structures reported in this paper have been deposited with the Cambridge Crystallographic Data Centre as supplementary publication no. CCDC-293541 (1-( $\alpha$-naphtol $\left.)_{2}\right)$, CCDC-293542 (1. $\beta$-naphtol), and CCDC-293543 (1-(DMF $\left.)_{2}\right)$. These data can be obtained free of charge via www.ccdc.cam.ac.uk/conts.retrieving.html.

Crystal data for 1·(DMF) $)_{2}: \mathrm{C}_{58} \mathrm{H}_{66} \mathrm{~N}_{6} \mathrm{O}_{14}, M=1071.19$, yellow prism, triclinic, space group $P-1$ (no. 2), $a=$

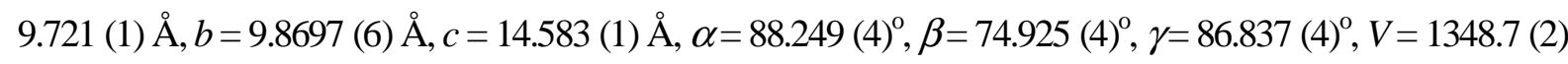
$\AA^{3}, T=113 K, Z=1, D_{\mathrm{c}}=1.319 \mathrm{~g} \mathrm{~cm}^{-1}, F(000)=568, \mu=0.95 \mathrm{~cm}^{-1}$, crystal dimensions $0.10 \times 0.10 \times 0.34$ $\mathrm{mm}^{3}, 11533$ reflections collected, 5931 unique $\left(R_{\mathrm{int}}=0.042\right)$ which were used in all calculations. $R_{1}=0.045$ for $I>2 \sigma(I), R_{1}=0.071, w R_{2}=0.114, S=1.270$ for all data. Anal. Calcd for $\mathrm{C}_{58} \mathrm{H}_{66} \mathrm{~N}_{6} \mathrm{O}_{14}: \mathrm{C}, 65.03 ; \mathrm{H}, 6.21 ; \mathrm{N}$, 7.85. Found: C, 65.02; H, 6.19; N, 7.77.

Crystal data for $1 \cdot(\alpha \text {-naphtol) })_{2}: \mathrm{C}_{72} \mathrm{H}_{68} \mathrm{~N}_{4} \mathrm{O}_{14}, M=1213.35$, yellow prism, monoclinic, space group $P 2_{1} / a$ (no.

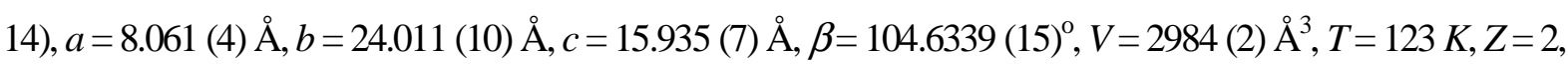
$D_{\mathrm{c}}=1.350 \mathrm{~g} \mathrm{~cm}^{-1}, F(000)=1280, \mu=0.95 \mathrm{~cm}^{-1}$, crystal dimensions $0.14 \times 0.08 \times 0.06 \mathrm{~mm}^{3}, 5593$ reflections collected, 908 unique $\left(R_{\mathrm{int}}=0.065\right)$ which were used in all calculations. $R_{1}=0.047$ for $I>2 \sigma(I), R_{1}=0.100$, $w R_{2}=0.129, S=1.003$ for all data. Anal. Calcd for $\mathrm{C}_{72} \mathrm{H}_{68} \mathrm{~N}_{4} \mathrm{O}_{14}: \mathrm{C}, 71.27 ; \mathrm{H}, 5.65 ; \mathrm{N}, 4.62$. Found: C, 71.11; 
H, 5.60, N; 4.63.

Crystal data for $1 \cdot \beta$-naphtol: $\mathrm{C}_{62} \mathrm{H}_{60} \mathrm{~N}_{4} \mathrm{O}_{13}, M=1069.18$, yellow prism, triclinic, space group $P-1$ (no. 2 ), $a=$

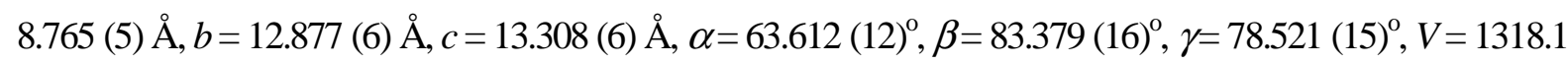
(11) $\AA^{3}, T=123 K, Z=1, D_{\mathrm{c}}=1.347 \mathrm{~g} \mathrm{~cm}^{-1}, F(000)=564, \mu=0.95 \mathrm{~cm}^{-1}$, crystal dimensions $0.15 \times 0.10 \times 0.08$ $\mathrm{mm}^{3}, 2486$ reflections collected, 804 unique $\left(R_{\mathrm{int}}=0.035\right)$ which were used in all calculations. $R_{1}=0.043$, for $I$ $>2 \sigma(I), R_{1}=0.059, w R_{2}=0.135, S=1.004$ for all data. Anal. Calcd for $\mathrm{C}_{62} \mathrm{H}_{60} \mathrm{~N}_{4} \mathrm{O}_{13}: \mathrm{C}, 69.65 ; \mathrm{H}, 5.66 ; \mathrm{N}$, 5.24. Found: C, 69.66; H, 5.59, N; 4.97.

(a)

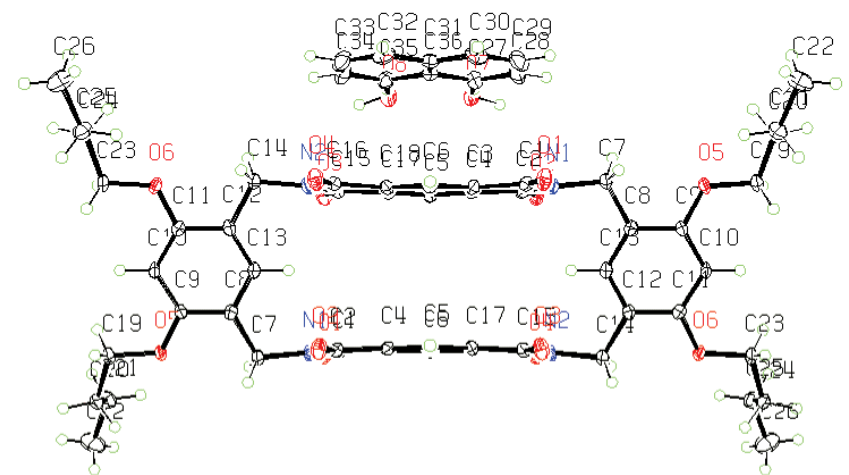

(b)

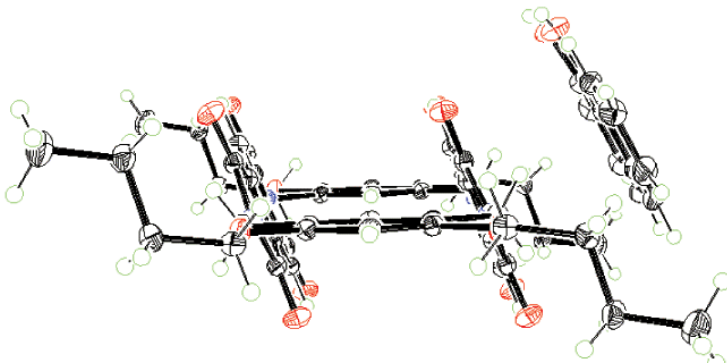

Figure S9. ORTEP drawings for 1·( $(\alpha \text {-naphtol })_{2}$. (a) Side view. (b) Bird-eye view. 
(a)

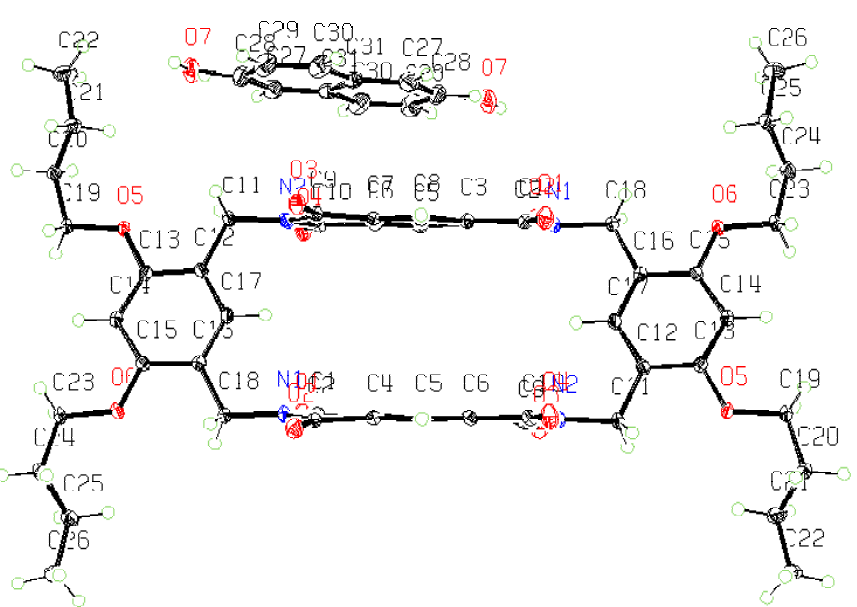

(b)

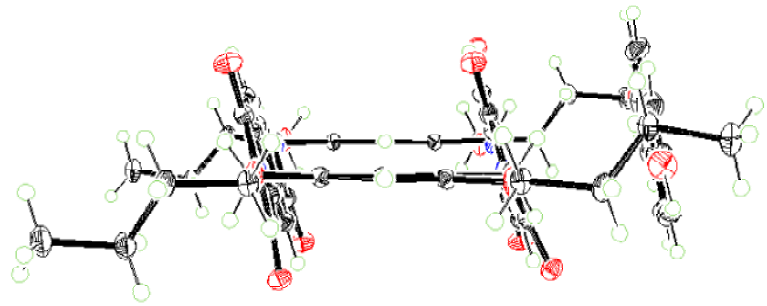

Figure S10. ORTEP drawings for $1 \cdot \beta$-naphtol. (a) Side view. (b) Bird-eye view.

\section{Electrochemistry}
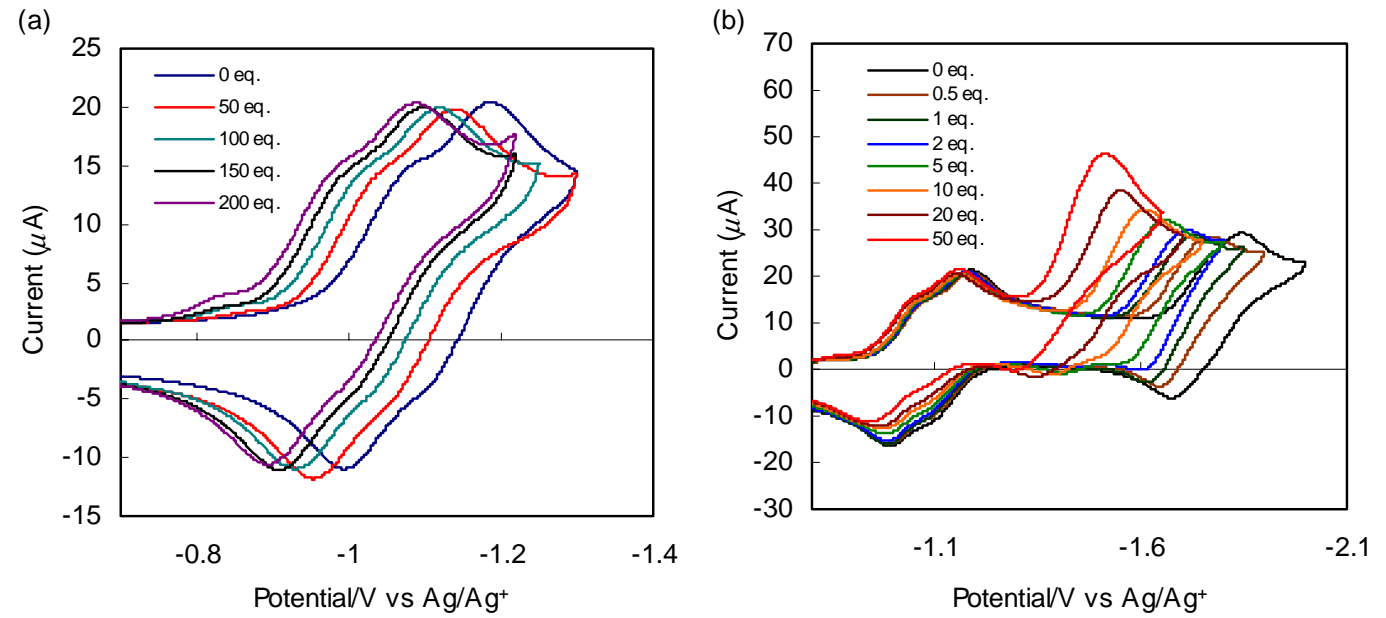

Figure S11. CV traces for (a) di-radical anion and (b) tetraanion of 1 in the presence of $\beta$-naphtol. 

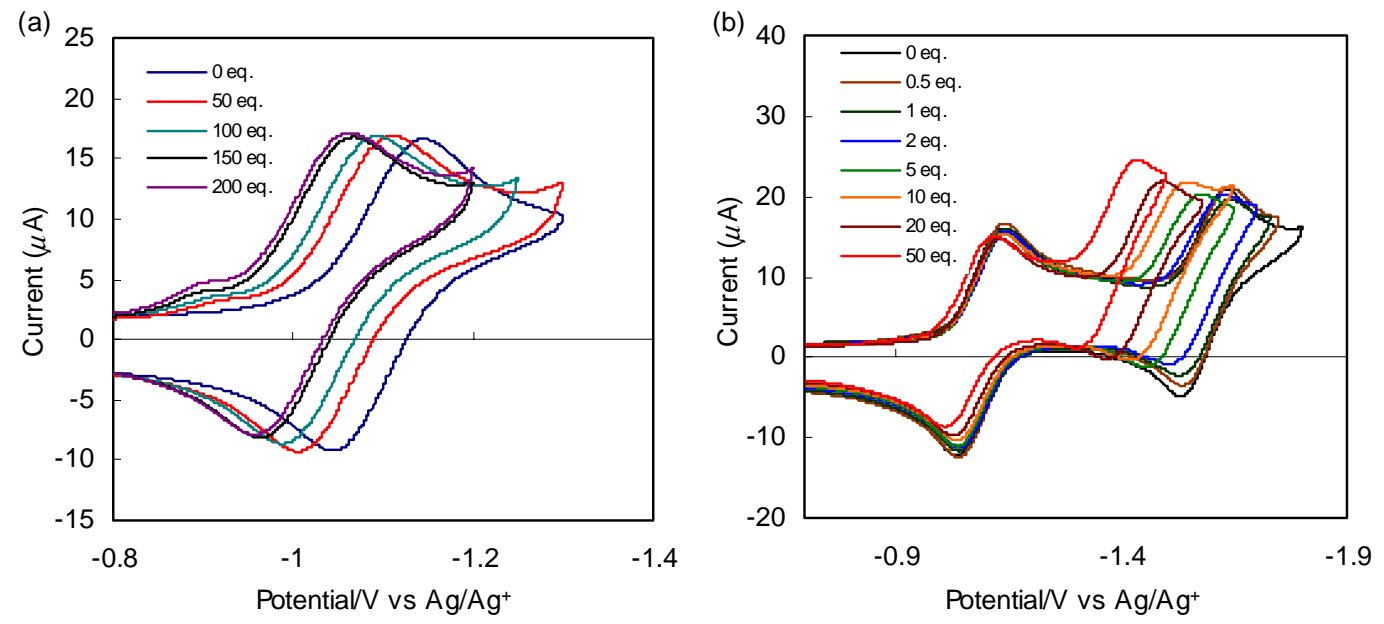

Figure S12. CV traces for (a) radical anion and (b) dianion of $\mathbf{2}$ in the presence of $\alpha$-naphtol.
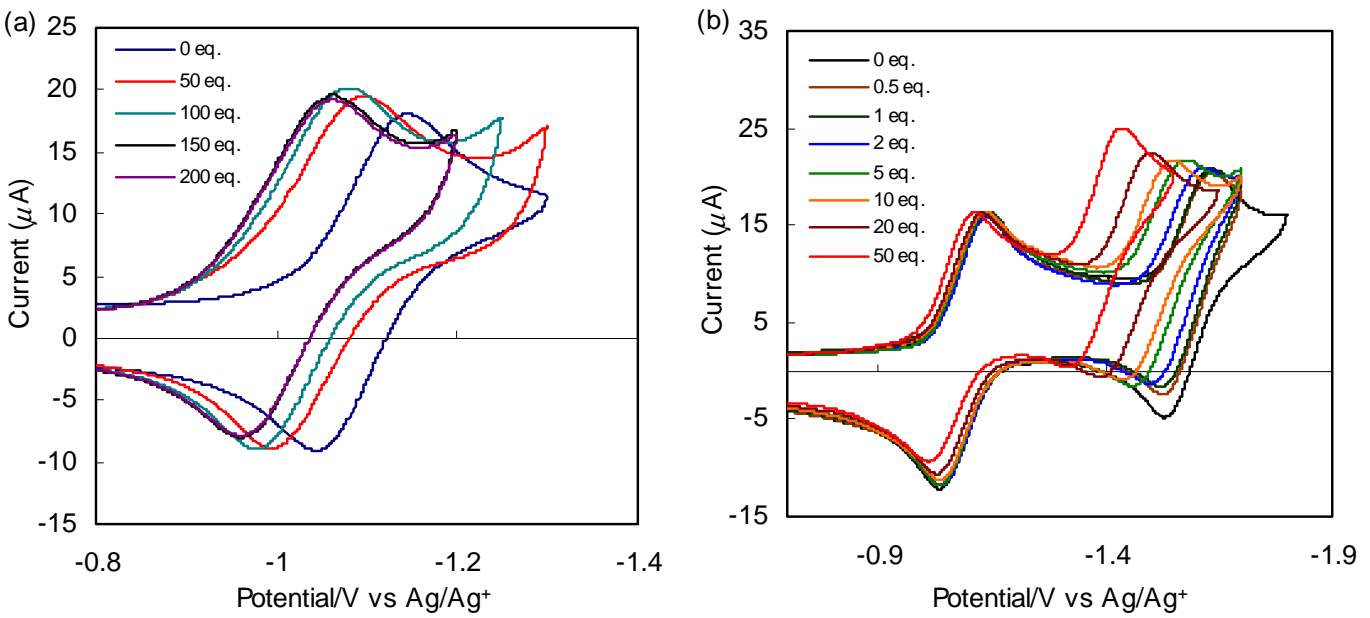

Figure S13. $\mathrm{CV}$ traces for (a) radical anion and (b) dianion of $\mathbf{2}$ in the presence of $\beta$-naphtol.

\section{Theoretical methods}

Table S1. Coordinates of syn-1'

\begin{tabular}{lccccc}
$\begin{array}{l}\text { Center } \\
\text { Number }\end{array}$ & $\begin{array}{c}\text { Atomic } \\
\text { Number }\end{array}$ & $\begin{array}{r}\text { Atomic } \\
\text { Type }\end{array}$ & X & \multicolumn{3}{c}{ Coordinates (Angstroms) } \\
\hline 1 & 6 & 0 & 6.864194 & 2.489903 & 2.923827 \\
2 & 8 & 0 & 6.289320 & 2.428421 & 1.628468 \\
3 & 6 & 0 & 6.864567 & -2.489450 & 2.924187 \\
4 & 8 & 0 & 6.289407 & -2.428265 & 1.628941 \\
5 & 6 & 0 & -6.863720 & 2.489947 & 2.924182 \\
6 & 8 & 0 & -6.289015 & 2.428519 & 1.628746 \\
& & & & & \\
& & & & &
\end{tabular}




\begin{tabular}{|c|c|c|c|c|c|}
\hline 7 & 6 & 0 & -6.864502 & -2.489420 & 2.924120 \\
\hline 8 & 8 & 0 & -6.289495 & -2.428165 & 1.628809 \\
\hline 9 & 8 & 0 & 3.015070 & -2.079542 & -3.012540 \\
\hline 10 & 8 & 0 & -3.015174 & -2.079424 & -3.012660 \\
\hline 11 & 8 & 0 & 3.018909 & -3.288974 & 1.431782 \\
\hline 12 & 8 & 0 & -3.019237 & -3.288933 & 1.431643 \\
\hline 13 & 8 & 0 & 3.015076 & 2.078265 & -3.012787 \\
\hline 14 & 8 & 0 & -3.015075 & 2.078278 & -3.012652 \\
\hline 15 & 6 & 0 & 1.173513 & -2.577188 & -1.485643 \\
\hline 16 & 6 & 0 & 1.174251 & -2.937658 & -0.132811 \\
\hline 17 & 6 & 0 & -0.000146 & -3.136659 & 0.587915 \\
\hline 18 & 6 & 0 & -2.598214 & 3.011122 & 0.327889 \\
\hline 19 & 6 & 0 & -1.174319 & 2.937873 & -0.133328 \\
\hline 20 & 6 & 0 & -1.173568 & 2.576728 & -1.485979 \\
\hline 21 & 6 & 0 & -2.595058 & 2.404527 & -1.920750 \\
\hline 22 & 6 & 0 & -2.595198 & -2.405180 & -1.920597 \\
\hline 23 & 6 & 0 & -1.173702 & -2.577174 & -1.485698 \\
\hline 24 & 6 & 0 & -1.174507 & -2.937640 & -0.132866 \\
\hline 25 & 6 & 0 & -2.598395 & -3.010612 & 0.328355 \\
\hline 26 & 7 & 0 & -3.377492 & 2.681902 & -0.791006 \\
\hline 27 & 7 & 0 & -3.377653 & -2.681932 & -0.790718 \\
\hline 28 & 6 & 0 & -4.837796 & 2.529336 & -0.760966 \\
\hline 29 & 6 & 0 & -4.837948 & -2.529312 & -0.760711 \\
\hline 30 & 6 & 0 & -6.321481 & 0.000182 & 1.706686 \\
\hline 31 & 6 & 0 & -5.970472 & 1.213294 & 1.101412 \\
\hline 32 & 6 & 0 & -5.273672 & 1.226522 & -0.121163 \\
\hline 33 & 6 & 0 & -4.957257 & 0.000010 & -0.707660 \\
\hline 34 & 6 & 0 & -5.273782 & -1.226413 & -0.121053 \\
\hline 35 & 6 & 0 & -5.970681 & -1.213017 & 1.101468 \\
\hline 36 & 8 & 0 & 3.019213 & 3.289897 & 1.430944 \\
\hline 37 & 8 & 0 & -3.019015 & 3.290088 & 1.431032 \\
\hline 38 & 6 & 0 & 4.957159 & -0.000178 & -0.707617 \\
\hline 39 & 6 & 0 & 5.970641 & 1.213178 & 1.101256 \\
\hline 40 & 6 & 0 & 6.321615 & 0.000086 & 1.706595 \\
\hline 41 & 6 & 0 & 5.970665 & -1.213135 & 1.101509 \\
\hline 42 & 6 & 0 & 5.273713 & 1.226357 & -0.121248 \\
\hline 43 & 6 & 0 & 5.273665 & -1.226578 & -0.120952 \\
\hline 44 & 6 & 0 & 4.837886 & 2.529152 & -0.761121 \\
\hline 45 & 6 & 0 & 4.837732 & -2.529503 & -0.760497 \\
\hline 46 & 6 & 0 & 2.598367 & 3.011136 & 0.327768 \\
\hline 47 & 7 & 0 & 3.377591 & 2.681787 & -0.791127 \\
\hline 48 & 6 & 0 & 2.595105 & 2.404367 & -1.920823 \\
\hline 49 & 6 & 0 & 2.595030 & -2.405196 & -1.920471 \\
\hline 50 & 7 & 0 & 3.377429 & -2.681990 & -0.790563 \\
\hline 51 & 6 & 0 & 2.598117 & -3.010672 & 0.328470 \\
\hline 52 & 6 & 0 & 0.000081 & 3.137242 & 0.587298 \\
\hline 53 & 6 & 0 & 1.174450 & 2.937845 & -0.133374 \\
\hline 54 & 6 & 0 & 1.173638 & 2.576682 & -1.486020 \\
\hline 55 & 6 & 0 & 0.000019 & 2.388475 & -2.210583 \\
\hline 56 & 6 & 0 & -0.000076 & -2.389289 & -2.210343 \\
\hline 57 & 1 & 0 & 6.217618 & 2.012815 & 3.670870 \\
\hline \multirow[t]{2}{*}{58} & 1 & 0 & 7.857679 & 2.022574 & 2.947494 \\
\hline & & & & $\mathrm{S} 12$ & \\
\hline
\end{tabular}




\begin{tabular}{|c|c|c|c|c|c|}
\hline 59 & 1 & 0 & 6.961143 & 3.551803 & 3.155474 \\
\hline 60 & 1 & 0 & 7.857991 & -2.021973 & 2.947558 \\
\hline 61 & 1 & 0 & 6.218090 & -2.012330 & 3.671295 \\
\hline 62 & 1 & 0 & 6.961735 & -3.551299 & 3.155980 \\
\hline 63 & 1 & 0 & -7.857253 & 2.022720 & 2.947929 \\
\hline 64 & 1 & 0 & -6.217104 & 2.012726 & 3.671103 \\
\hline 65 & 1 & 0 & -6.960524 & 3.551838 & 3.155937 \\
\hline 66 & 1 & 0 & -6.217869 & -2.012464 & 3.671196 \\
\hline 67 & 1 & 0 & -7.857866 & -2.021826 & 2.947664 \\
\hline 68 & 1 & 0 & -6.961785 & -3.551282 & 3.155804 \\
\hline 69 & 1 & 0 & -0.000171 & -3.408979 & 1.638338 \\
\hline 70 & 1 & 0 & -5.158415 & 2.583241 & -1.804678 \\
\hline 71 & 1 & 0 & -5.251454 & 3.378070 & -0.216710 \\
\hline 72 & 1 & 0 & -5.251654 & -3.377975 & -0.216382 \\
\hline 73 & 1 & 0 & -5.158558 & -2.583334 & -1.804422 \\
\hline 74 & 1 & 0 & -6.863764 & 0.000248 & 2.641511 \\
\hline 75 & 1 & 0 & -4.428143 & -0.000059 & -1.658851 \\
\hline 76 & 1 & 0 & 4.427967 & -0.000286 & -1.658764 \\
\hline 77 & 1 & 0 & 6.863993 & 0.000189 & 2.641363 \\
\hline 78 & 1 & 0 & 5.158477 & 2.582971 & -1.804847 \\
\hline 79 & 1 & 0 & 5.251607 & 3.377901 & -0.216938 \\
\hline 80 & 1 & 0 & 5.251319 & -3.378144 & -0.216042 \\
\hline 81 & 1 & 0 & 5.158405 & -2.583671 & -1.804181 \\
\hline 82 & 1 & 0 & 0.000104 & 3.410065 & 1.637590 \\
\hline 83 & 1 & 0 & -0.000005 & 2.099636 & -3.256621 \\
\hline 84 & 1 & 0 & -0.000051 & -2.101007 & -3.256534 \\
\hline
\end{tabular}

Zero-point correction $=0.630901$ (Hartree/Particle)

Sum of electronic and zero-point Energies $=-2662.286635$

Table S2. Coordinates of anti-1'

\begin{tabular}{ccrrrr}
$\begin{array}{l}\text { Center } \\
\text { Number }\end{array}$ & $\begin{array}{c}\text { Atomic } \\
\text { Number }\end{array}$ & \multicolumn{2}{c}{$\begin{array}{c}\text { Atomic } \\
\text { Type }\end{array}$} & X & Coordinates (Angstroms) \\
\hline 1 & 6 & 0 & -7.766843 & -2.487824 & 1.504733 \\
2 & 8 & 0 & -6.815441 & -2.428244 & 0.453788 \\
3 & 6 & 0 & -7.768779 & 2.485286 & 1.505668 \\
4 & 8 & 0 & -6.816257 & 2.426892 & 0.455682 \\
5 & 6 & 0 & 7.767632 & -2.487527 & -1.503988 \\
6 & 8 & 0 & 6.815364 & -2.427999 & -0.453842 \\
7 & 6 & 0 & 7.768174 & 2.485477 & -1.506338 \\
8 & 8 & 0 & 6.815921 & 2.427134 & -0.456087 \\
9 & 8 & 0 & -2.278827 & 2.596327 & -3.032402 \\
10 & 8 & 0 & 3.529475 & 2.738877 & -1.399450 \\
11 & 8 & 0 & -3.529752 & 2.740105 & 1.399135 \\
12 & 8 & 0 & 2.278623 & 2.598201 & 3.032220 \\
13 & 8 & 0 & -2.277168 & -2.597194 & -3.033202 \\
14 & 8 & 0 & 3.530367 & -2.739471 & -1.397535 \\
15 & 6 & 0 & -0.940648 & 2.715020 & -0.991424 \\
16 & 6 & 0 & -1.320145 & 2.726440 & 0.356061 \\
& & & & S13 &
\end{tabular}




\begin{tabular}{|c|c|c|c|c|c|}
\hline 17 & 6 & 0 & -0.392203 & 2.726339 & 1.393772 \\
\hline 18 & 6 & 0 & 2.183058 & -2.651592 & 1.823922 \\
\hline 19 & 6 & 0 & 0.940228 & -2.714846 & 0.991890 \\
\hline 20 & 6 & 0 & 1.320339 & -2.726056 & -0.355416 \\
\hline 21 & 6 & 0 & 2.816881 & -2.708133 & -0.417235 \\
\hline 22 & 6 & 0 & 2.816431 & 2.708071 & -0.418806 \\
\hline 23 & 6 & 0 & 1.319918 & 2.726095 & -0.356314 \\
\hline 24 & 6 & 0 & 0.940426 & 2.715631 & 0.991176 \\
\hline 25 & 6 & 0 & 2.183645 & 2.652827 & 1.822676 \\
\hline 26 & 7 & 0 & 3.251606 & -2.649071 & 0.915861 \\
\hline 27 & 7 & 0 & 3.251761 & 2.649661 & 0.914102 \\
\hline 28 & 6 & 0 & 4.658623 & -2.530397 & 1.317839 \\
\hline 29 & 6 & 0 & 4.659011 & 2.531603 & 1.315394 \\
\hline 30 & 6 & 0 & 6.875305 & -0.000481 & -0.511876 \\
\hline 31 & 6 & 0 & 6.343035 & -1.212681 & -0.057337 \\
\hline 32 & 6 & 0 & 5.282356 & -1.225989 & 0.866787 \\
\hline 33 & 6 & 0 & 4.790230 & 0.000587 & 1.313944 \\
\hline 34 & 6 & 0 & 5.282595 & 1.226685 & 0.865572 \\
\hline 35 & 6 & 0 & 6.343262 & 1.212294 & -0.058462 \\
\hline 36 & 8 & 0 & -3.530079 & -2.738755 & 1.397867 \\
\hline 37 & 8 & 0 & 2.277446 & -2.596199 & 3.033483 \\
\hline 38 & 6 & 0 & -4.790163 & 0.000356 & -1.313863 \\
\hline 39 & 6 & 0 & -6.343062 & -1.212935 & 0.057283 \\
\hline 40 & 6 & 0 & -6.875507 & -0.000718 & 0.511644 \\
\hline 41 & 6 & 0 & -6.343496 & 1.212041 & 0.058226 \\
\hline 42 & 6 & 0 & -5.282268 & -1.226243 & -0.866654 \\
\hline 43 & 6 & 0 & -5.282680 & 1.226434 & -0.865686 \\
\hline 44 & 6 & 0 & -4.658339 & -2.530629 & -1.317511 \\
\hline 45 & 6 & 0 & -4.659229 & 2.531407 & -1.315554 \\
\hline 46 & 6 & 0 & -2.816591 & -2.707710 & 0.417552 \\
\hline 47 & 7 & 0 & -3.251306 & -2.649034 & -0.915544 \\
\hline 48 & 6 & 0 & -2.182760 & -2.652100 & -1.823627 \\
\hline 49 & 6 & 0 & -2.183864 & 2.651841 & -1.822888 \\
\hline 50 & 7 & 0 & -3.251987 & 2.649635 & -0.914347 \\
\hline 51 & 6 & 0 & -2.816663 & 2.708626 & 0.418550 \\
\hline 52 & 6 & 0 & -0.392584 & -2.725246 & 1.393883 \\
\hline 53 & 6 & 0 & -1.320048 & -2.725799 & 0.355748 \\
\hline 54 & 6 & 0 & -0.939934 & -2.715095 & -0.991566 \\
\hline 55 & 6 & 0 & 0.392875 & -2.725758 & -1.393556 \\
\hline 56 & 6 & 0 & 0.391979 & 2.725347 & -1.394025 \\
\hline 57 & 1 & 0 & -7.387513 & -2.005236 & 2.413883 \\
\hline 58 & 1 & 0 & -8.719506 & -2.025414 & 1.213978 \\
\hline 59 & 1 & 0 & -7.928857 & -3.549408 & 1.698971 \\
\hline 60 & 1 & 0 & -8.720840 & 2.022429 & 1.213666 \\
\hline 61 & 1 & 0 & -7.390104 & 2.002437 & 2.414960 \\
\hline 62 & 1 & 0 & -7.931649 & 3.546660 & 1.700334 \\
\hline 63 & 1 & 0 & 7.388902 & -2.005281 & -2.413572 \\
\hline 64 & 1 & 0 & 8.719908 & -2.024736 & -1.212571 \\
\hline 65 & 1 & 0 & 7.930144 & -3.549104 & -1.697853 \\
\hline 66 & 1 & 0 & 8.720275 & 2.022538 & -1.214604 \\
\hline 67 & 1 & 0 & 7.389191 & 2.002688 & -2.415529 \\
\hline \multirow[t]{2}{*}{68} & 1 & 0 & 7.931090 & 3.546844 & -1.700998 \\
\hline & & & & S14 & \\
\hline
\end{tabular}




\begin{tabular}{|c|c|c|c|c|c|}
\hline 69 & 1 & 0 & -0.685977 & 2.720072 & 2.438400 \\
\hline 70 & 1 & 0 & 5.210215 & -3.374500 & 0.902662 \\
\hline 71 & 1 & 0 & 4.652006 & -2.617239 & 2.407935 \\
\hline 72 & 1 & 0 & 4.653089 & 2.619824 & 2.405384 \\
\hline 73 & 1 & 0 & 5.210254 & 3.375229 & 0.898783 \\
\hline 74 & 1 & 0 & 7.693009 & -0.000892 & -1.218616 \\
\hline 75 & 1 & 0 & 3.969640 & 0.001043 & 2.029850 \\
\hline 76 & 1 & 0 & -3.969465 & 0.000785 & -2.029643 \\
\hline 77 & 1 & 0 & -7.693441 & -0.001157 & 1.218114 \\
\hline 78 & 1 & 0 & -4.651755 & -2.617676 & -2.407590 \\
\hline 79 & 1 & 0 & -5.209813 & -3.374737 & -0.902173 \\
\hline 80 & 1 & 0 & -5.210522 & 3.374959 & -0.898863 \\
\hline 81 & 1 & 0 & -4.653411 & 2.619647 & -2.405541 \\
\hline 82 & 1 & 0 & -0.686835 & -2.718406 & 2.438373 \\
\hline 83 & 1 & 0 & 0.687130 & -2.719323 & -2.438047 \\
\hline 84 & 1 & 0 & 0.685757 & 2.718357 & -2.438648 \\
\hline
\end{tabular}

Zero-point correction $=0.630829$ (Hartree/Particle)

Sum of electronic and zero-point Energies $=-2662.284863$

Table S3. Coordinates of parallel-1'

\begin{tabular}{|c|c|c|c|c|c|}
\hline \multirow{2}{*}{$\begin{array}{l}\text { Center } \\
\text { Number }\end{array}$} & \multirow{2}{*}{$\begin{array}{l}\text { Atomic } \\
\text { Number }\end{array}$} & \multirow{2}{*}{$\begin{array}{l}\text { Atomic } \\
\text { Type }\end{array}$} & \multicolumn{3}{|c|}{ Coordinates (Angstroms) } \\
\hline & & & $\mathrm{X}$ & $\mathrm{Y}$ & $\mathrm{Z}$ \\
\hline 1 & 8 & 0 & -3.017447 & 2.567790 & -2.302272 \\
\hline 2 & 8 & 0 & -3.018015 & 2.563168 & 2.303083 \\
\hline 3 & 8 & 0 & 3.019602 & 2.567992 & -2.301559 \\
\hline 4 & 8 & 0 & 3.019053 & 2.563543 & 2.303823 \\
\hline 5 & 8 & 0 & 7.529036 & 2.437347 & 0.000135 \\
\hline 6 & 8 & 0 & 7.527530 & -2.438906 & -0.001118 \\
\hline 7 & 7 & 0 & -3.378333 & 2.506814 & 0.000309 \\
\hline 8 & 7 & 0 & 3.379914 & 2.507935 & 0.001123 \\
\hline 9 & 6 & 0 & -2.595865 & 2.528250 & -1.166109 \\
\hline 10 & 6 & 0 & -2.596137 & 2.525911 & 1.166951 \\
\hline 11 & 6 & 0 & -1.173150 & 2.512570 & -0.699580 \\
\hline 12 & 6 & 0 & -1.173319 & 2.511190 & 0.700733 \\
\hline 13 & 6 & 0 & 0.000625 & 2.513451 & 1.449058 \\
\hline 14 & 6 & 0 & 1.174746 & 2.511421 & 0.701017 \\
\hline 15 & 6 & 0 & 1.174916 & 2.512775 & -0.699303 \\
\hline 16 & 6 & 0 & 0.000972 & 2.516268 & -1.447614 \\
\hline 17 & 6 & 0 & 2.597740 & 2.528675 & -1.165491 \\
\hline 18 & 6 & 0 & 2.597458 & 2.526439 & 1.167576 \\
\hline 19 & 6 & 0 & 4.834033 & 2.572108 & 0.001349 \\
\hline 20 & 6 & 0 & 5.529172 & 1.221878 & 0.000614 \\
\hline 21 & 6 & 0 & 6.932834 & 1.206175 & 0.000032 \\
\hline 22 & 6 & 0 & 7.639695 & -0.000809 & -0.000588 \\
\hline 23 & 6 & 0 & 6.932094 & -1.207362 & -0.000602 \\
\hline 24 & 6 & 0 & 5.528431 & -1.222201 & -0.000019 \\
\hline 25 & 6 & 0 & 4.859223 & 0.000047 & 0.000581 \\
\hline \multirow[t]{2}{*}{26} & 6 & 0 & 4.832418 & -2.571957 & 0.000032 \\
\hline & & & & $\mathrm{S} 15$ & \\
\hline
\end{tabular}




\begin{tabular}{|c|c|c|c|c|c|}
\hline 27 & 6 & 0 & 8.945798 & 2.511998 & -0.000239 \\
\hline 28 & 6 & 0 & 8.944255 & -2.514430 & -0.001652 \\
\hline 29 & 8 & 0 & 3.017455 & -2.567977 & 2.302520 \\
\hline 30 & 8 & 0 & 3.018000 & -2.562975 & -2.302831 \\
\hline 31 & 8 & 0 & -3.019583 & -2.568196 & 2.301851 \\
\hline 32 & 8 & 0 & -3.019074 & -2.563375 & -2.303531 \\
\hline 33 & 8 & 0 & -7.529032 & -2.437366 & -0.000628 \\
\hline 34 & 8 & 0 & -7.527526 & 2.438885 & 0.000562 \\
\hline 35 & 7 & 0 & 3.378329 & -2.506774 & -0.000057 \\
\hline 36 & 7 & 0 & -3.379914 & -2.507941 & -0.000824 \\
\hline 37 & 6 & 0 & 2.595868 & -2.528255 & 1.166365 \\
\hline 38 & 6 & 0 & 2.596128 & -2.525730 & -1.166697 \\
\hline 39 & 6 & 0 & 1.173152 & -2.512597 & 0.699845 \\
\hline 40 & 6 & 0 & 1.173311 & -2.511096 & -0.700468 \\
\hline 41 & 6 & 0 & -0.000637 & -2.513324 & -1.448786 \\
\hline 42 & 6 & 0 & -1.174753 & -2.511362 & -0.700737 \\
\hline 43 & 6 & 0 & -1.174912 & -2.512834 & 0.699584 \\
\hline 44 & 6 & 0 & -0.000964 & -2.516391 & 1.447887 \\
\hline 45 & 6 & 0 & -2.597731 & -2.528744 & 1.165781 \\
\hline 46 & 6 & 0 & -2.597468 & -2.526352 & -1.167286 \\
\hline 47 & 6 & 0 & -4.834032 & -2.572126 & -0.001047 \\
\hline 48 & 6 & 0 & -5.529169 & -1.221896 & -0.000560 \\
\hline 49 & 6 & 0 & -6.932831 & -1.206195 & -0.000386 \\
\hline 50 & 6 & 0 & -7.639692 & 0.000789 & 0.000010 \\
\hline 51 & 6 & 0 & -6.932090 & 1.207342 & 0.000217 \\
\hline 52 & 6 & 0 & -5.528426 & 1.222183 & 0.000042 \\
\hline 53 & 6 & 0 & -4.859218 & -0.000065 & -0.000342 \\
\hline 54 & 6 & 0 & -4.832424 & 2.571948 & 0.000204 \\
\hline 55 & 6 & 0 & -8.945795 & -2.512018 & -0.000768 \\
\hline 56 & 6 & 0 & -8.944251 & 2.514410 & 0.000593 \\
\hline 57 & 1 & 0 & 0.000514 & 2.519039 & 2.534214 \\
\hline 58 & 1 & 0 & 0.001118 & 2.523944 & -2.532794 \\
\hline 59 & 1 & 0 & 5.129634 & 3.146617 & 0.884504 \\
\hline 60 & 1 & 0 & 5.129834 & 3.147672 & -0.881041 \\
\hline 61 & 1 & 0 & 8.720660 & -0.001125 & -0.001023 \\
\hline 62 & 1 & 0 & 3.774464 & 0.000334 & 0.001012 \\
\hline 63 & 1 & 0 & 5.127874 & -3.147286 & -0.882655 \\
\hline 64 & 1 & 0 & 5.127592 & -3.147106 & 0.882915 \\
\hline 65 & 1 & 0 & 9.373137 & 2.042941 & -0.896142 \\
\hline 66 & 1 & 0 & 9.373648 & 2.042318 & 0.895098 \\
\hline 67 & 1 & 0 & 9.188987 & 3.575940 & 0.000051 \\
\hline 68 & 1 & 0 & 9.186773 & -3.578507 & -0.002057 \\
\hline 69 & 1 & 0 & 9.371814 & -2.045076 & -0.897328 \\
\hline 70 & 1 & 0 & 9.372453 & -2.045554 & 0.893930 \\
\hline 71 & 1 & 0 & -0.000533 & -2.518818 & -2.533943 \\
\hline 72 & 1 & 0 & -0.001103 & -2.524148 & 2.533066 \\
\hline 73 & 1 & 0 & -5.129646 & -3.146793 & -0.884093 \\
\hline 74 & 1 & 0 & -5.129815 & -3.147532 & 0.881453 \\
\hline 75 & 1 & 0 & -8.720656 & 0.001107 & 0.000134 \\
\hline 76 & 1 & 0 & -3.774460 & -0.000354 & -0.000467 \\
\hline 77 & 1 & 0 & -5.127888 & 3.147145 & 0.882976 \\
\hline \multirow[t]{2}{*}{78} & 1 & 0 & -5.127614 & 3.147222 & -0.882592 \\
\hline & & & & $\mathrm{S} 16$ & \\
\hline
\end{tabular}




\begin{tabular}{rrrrrr}
79 & 1 & 0 & -9.373459 & -2.042960 & 0.894979 \\
80 & 1 & 0 & -9.373319 & -2.042340 & -0.896261 \\
81 & 1 & 0 & -9.188983 & -3.575960 & -0.001142 \\
82 & 1 & 0 & -9.186768 & 3.578487 & 0.000890 \\
83 & 1 & 0 & -9.372127 & 2.045077 & 0.896127 \\
84 & 1 & 0 & -9.372131 & 2.045515 & -0.895131 \\
\hline
\end{tabular}

Zero-point correction $=0.630062$ (Hartree/Particle)

Sum of electronic and zero-point Energies $=-2662.285653$

Table S4. Coordinates of 1

\begin{tabular}{|c|c|c|c|c|c|}
\hline \multirow{2}{*}{$\begin{array}{l}\text { Center } \\
\text { Number }\end{array}$} & \multirow{2}{*}{$\begin{array}{l}\text { Atomic } \\
\text { Number }\end{array}$} & \multirow{2}{*}{$\begin{array}{c}\text { Atomic } \\
\text { Type }\end{array}$} & \multicolumn{3}{|c|}{ Coordinates (Angstroms) } \\
\hline & & & $\mathrm{X}$ & $\mathrm{Y}$ & $\mathrm{Z}$ \\
\hline 1 & 8 & 0 & -3.015840 & -2.981340 & 1.756130 \\
\hline 2 & 8 & 0 & -2.748990 & -2.354980 & -2.798580 \\
\hline 3 & 8 & 0 & 3.003080 & -2.724510 & 2.144370 \\
\hline 4 & 8 & 0 & 3.269690 & -2.104300 & -2.411200 \\
\hline 5 & 8 & 0 & 7.630480 & -2.090780 & 0.113250 \\
\hline 6 & 8 & 0 & 7.379970 & 2.745910 & 0.745750 \\
\hline 7 & 7 & 0 & -3.245310 & -2.624410 & -0.536430 \\
\hline 8 & 7 & 0 & 3.493210 & -2.339820 & -0.102430 \\
\hline 9 & 6 & 0 & -2.531820 & -2.769370 & 0.664680 \\
\hline 10 & 6 & 0 & -2.396660 & -2.452700 & -1.642460 \\
\hline 11 & 6 & 0 & -1.087050 & -2.629310 & 0.297310 \\
\hline 12 & 6 & 0 & -1.005900 & -2.439860 & -1.087830 \\
\hline 13 & 6 & 0 & 0.208310 & -2.292750 & -1.752700 \\
\hline 14 & 6 & 0 & 1.335260 & -2.341680 & -0.936810 \\
\hline 15 & 6 & 0 & 1.254190 & -2.530740 & 0.448390 \\
\hline 16 & 6 & 0 & 0.040230 & -2.683980 & 1.112430 \\
\hline 17 & 6 & 0 & 2.646740 & -2.549790 & 0.998580 \\
\hline 18 & 6 & 0 & 2.781640 & -2.235470 & -1.308960 \\
\hline 19 & 6 & 0 & 4.946570 & -2.343690 & -0.018730 \\
\hline 20 & 6 & 0 & 5.574150 & -0.975890 & 0.183280 \\
\hline 21 & 6 & 0 & 6.975080 & -0.898290 & 0.244310 \\
\hline 22 & 6 & 0 & 7.620650 & 0.329520 & 0.422840 \\
\hline 23 & 6 & 0 & 6.857550 & 1.496370 & 0.546820 \\
\hline 24 & 6 & 0 & 5.455270 & 1.445680 & 0.490260 \\
\hline 25 & 6 & 0 & 4.846400 & 0.204890 & 0.309590 \\
\hline 26 & 6 & 0 & 4.692180 & 2.750360 & 0.638320 \\
\hline 27 & 6 & 0 & 9.056640 & -2.118170 & 0.156710 \\
\hline 28 & 6 & 0 & 9.500020 & -3.564910 & -0.011960 \\
\hline 29 & 6 & 0 & 11.025960 & -3.720470 & 0.019430 \\
\hline 30 & 6 & 0 & 11.476240 & -5.174670 & -0.152880 \\
\hline 31 & 6 & 0 & 8.796510 & 2.953430 & 0.773800 \\
\hline 32 & 6 & 0 & 9.421380 & 3.030440 & -0.621420 \\
\hline 33 & 6 & 0 & 8.836680 & 4.129270 & -1.524490 \\
\hline 34 & 6 & 0 & 9.080310 & 5.558340 & -1.025960 \\
\hline 35 & 8 & 0 & 3.015480 & 2.981470 & -1.755310 \\
\hline \multirow[t]{2}{*}{36} & 8 & 0 & 2.749610 & 2.354710 & 2.799400 \\
\hline & & & & S17 & \\
\hline
\end{tabular}




\begin{tabular}{|c|c|c|c|c|c|}
\hline 37 & 8 & 0 & -3.003540 & 2.724520 & -2.142300 \\
\hline 38 & 8 & 0 & -3.269130 & 2.103840 & 2.413270 \\
\hline 39 & 8 & 0 & -7.630330 & 2.090790 & -0.113250 \\
\hline 40 & 8 & 0 & -7.379750 & -2.745670 & -0.747570 \\
\hline 41 & 7 & 0 & 3.245440 & 2.624360 & 0.537170 \\
\hline 42 & 7 & 0 & -3.493160 & 2.339590 & 0.104570 \\
\hline 43 & 6 & 0 & 2.531680 & 2.769370 & -0.663790 \\
\hline 44 & 6 & 0 & 2.397040 & 2.452590 & 1.643370 \\
\hline 45 & 6 & 0 & 1.087000 & 2.629260 & -0.296120 \\
\hline 46 & 6 & 0 & 1.006160 & 2.439710 & 1.089030 \\
\hline 47 & 6 & 0 & -0.207910 & 2.292490 & 1.754140 \\
\hline 48 & 6 & 0 & -1.335040 & 2.341460 & 0.938480 \\
\hline 49 & 6 & 0 & -1.254260 & 2.530630 & -0.446710 \\
\hline 50 & 6 & 0 & -0.040440 & 2.683960 & -1.111000 \\
\hline 51 & 6 & 0 & -2.646930 & 2.549720 & -0.996600 \\
\hline 52 & 6 & 0 & -2.781330 & 2.235190 & 1.310940 \\
\hline 53 & 6 & 0 & -4.946540 & 2.343390 & 0.021170 \\
\hline 54 & 6 & 0 & -5.574040 & 0.975740 & -0.182080 \\
\hline 55 & 6 & 0 & -6.974930 & 0.898290 & -0.244340 \\
\hline 56 & 6 & 0 & -7.620470 & -0.329380 & -0.423940 \\
\hline 57 & 6 & 0 & -6.857370 & -1.496250 & -0.547740 \\
\hline 58 & 6 & 0 & -5.455130 & -1.445710 & -0.489990 \\
\hline 59 & 6 & 0 & -4.846300 & -0.205060 & -0.308250 \\
\hline 60 & 6 & 0 & -4.692040 & -2.750400 & -0.637910 \\
\hline 61 & 6 & 0 & -9.056430 & 2.118380 & -0.158080 \\
\hline 62 & 6 & 0 & -9.499780 & 3.565100 & 0.010820 \\
\hline 63 & 6 & 0 & -11.025660 & 3.720920 & -0.021890 \\
\hline 64 & 6 & 0 & -11.475870 & 5.175110 & 0.150690 \\
\hline 65 & 6 & 0 & -8.796290 & -2.953030 & -0.776700 \\
\hline 66 & 6 & 0 & -9.422170 & -3.030250 & 0.618050 \\
\hline 67 & 6 & 0 & -8.838240 & -4.129320 & 1.521310 \\
\hline 68 & 6 & 0 & -9.081630 & -5.558260 & 1.022320 \\
\hline 69 & 1 & 0 & 0.271930 & -2.153750 & -2.827080 \\
\hline 70 & 1 & 0 & -0.022670 & -2.837940 & 2.184840 \\
\hline 71 & 1 & 0 & 5.320570 & -2.795200 & -0.942570 \\
\hline 72 & 1 & 0 & 5.217460 & -3.009120 & 0.806730 \\
\hline 73 & 1 & 0 & 8.699590 & 0.373090 & 0.460660 \\
\hline 74 & 1 & 0 & 3.763510 & 0.157010 & 0.265130 \\
\hline 75 & 1 & 0 & 4.906740 & 3.204400 & 1.610630 \\
\hline 76 & 1 & 0 & 5.006900 & 3.459650 & -0.133060 \\
\hline 77 & 1 & 0 & 9.407800 & -1.711940 & 1.116690 \\
\hline 78 & 1 & 0 & 9.465640 & -1.488930 & -0.647510 \\
\hline 79 & 1 & 0 & 9.044090 & -4.167040 & 0.784520 \\
\hline 80 & 1 & 0 & 9.102360 & -3.945220 & -0.961800 \\
\hline 81 & 1 & 0 & 11.414870 & -3.328280 & 0.969780 \\
\hline 82 & 1 & 0 & 11.473470 & -3.103480 & -0.772570 \\
\hline 83 & 1 & 0 & 11.072620 & -5.811060 & 0.643740 \\
\hline 84 & 1 & 0 & 11.133130 & -5.584310 & -1.110490 \\
\hline 85 & 1 & 0 & 12.568270 & -5.258100 & -0.125950 \\
\hline 86 & 1 & 0 & 8.914740 & 3.902930 & 1.302980 \\
\hline 87 & 1 & 0 & 9.278860 & 2.179440 & 1.384180 \\
\hline \multirow[t]{2}{*}{88} & 1 & 0 & 9.313830 & 2.060080 & -1.121180 \\
\hline & & & & S18 & \\
\hline
\end{tabular}




\begin{tabular}{|c|c|c|c|c|c|}
\hline 89 & 1 & 0 & 10.500680 & 3.194690 & -0.486260 \\
\hline 90 & 1 & 0 & 7.759890 & 3.958770 & -1.643680 \\
\hline 91 & 1 & 0 & 9.275980 & 4.017690 & -2.524060 \\
\hline 92 & 1 & 0 & 8.697170 & 6.291380 & -1.744410 \\
\hline 93 & 1 & 0 & 10.151200 & 5.755910 & -0.889200 \\
\hline 94 & 1 & 0 & 8.580500 & 5.750760 & -0.069690 \\
\hline 95 & 1 & 0 & -0.271320 & 2.153420 & 2.828520 \\
\hline 96 & 1 & 0 & 0.022220 & 2.838010 & -2.183410 \\
\hline 97 & 1 & 0 & -5.320310 & 2.793950 & 0.945570 \\
\hline 98 & 1 & 0 & -5.217750 & 3.009610 & -0.803540 \\
\hline 99 & 1 & 0 & -8.699380 & -0.372830 & -0.462710 \\
\hline 100 & 1 & 0 & -3.763440 & -0.157300 & -0.262860 \\
\hline 101 & 1 & 0 & -4.906390 & -3.204370 & -1.610280 \\
\hline 102 & 1 & 0 & -5.006930 & -3.459730 & 0.133380 \\
\hline 103 & 1 & 0 & -9.406730 & 1.712610 & -1.118580 \\
\hline 104 & 1 & 0 & -9.466300 & 1.488840 & 0.645470 \\
\hline 105 & 1 & 0 & -9.043040 & 4.167510 & -0.784990 \\
\hline 106 & 1 & 0 & -9.102920 & 3.944940 & 0.961180 \\
\hline 107 & 1 & 0 & -11.413780 & 3.329220 & -0.972770 \\
\hline 108 & 1 & 0 & -11.473980 & 3.103630 & 0.769420 \\
\hline 109 & 1 & 0 & -11.071440 & 5.811790 & -0.645290 \\
\hline 110 & 1 & 0 & -11.133540 & 5.584260 & 1.108780 \\
\hline 111 & 1 & 0 & -12.567860 & 5.258720 & 0.122830 \\
\hline 112 & 1 & 0 & -8.914240 & -3.902420 & -1.306160 \\
\hline 113 & 1 & 0 & -9.278100 & -2.178860 & -1.387280 \\
\hline 114 & 1 & 0 & -9.314870 & -2.059990 & 1.118070 \\
\hline 115 & 1 & 0 & -10.501400 & -3.194350 & 0.482090 \\
\hline 116 & 1 & 0 & -7.761510 & -3.958940 & 1.641310 \\
\hline 117 & 1 & 0 & -9.278240 & -4.017910 & 2.520600 \\
\hline 118 & 1 & 0 & -8.699110 & -6.291490 & 1.740900 \\
\hline 119 & 1 & 0 & -10.152440 & -5.755700 & 0.884720 \\
\hline 120 & 1 & 0 & -8.581120 & -5.750540 & 0.066380 \\
\hline
\end{tabular}

Table S5. Coordinates of 2

\begin{tabular}{cccccc}
$\begin{array}{c}\text { Center } \\
\text { Number }\end{array}$ & $\begin{array}{c}\text { Atomic } \\
\text { Number }\end{array}$ & $\begin{array}{c}\text { Atomic } \\
\text { Type }\end{array}$ & $\mathrm{X}$ & \multicolumn{3}{c}{ Coordinates (Angstroms) } \\
\hline 1 & 6 & 0 & 1.139758 & 0.754042 & -1.296029 \\
2 & 6 & 0 & 1.205835 & -0.644162 & -1.295419 \\
3 & 6 & 0 & 0.068493 & -1.446754 & -1.301077 \\
4 & 6 & 0 & -1.139826 & -0.755658 & -1.295089 \\
5 & 6 & 0 & -1.205903 & 0.642546 & -1.296284 \\
6 & 6 & 0 & -0.068561 & 1.445130 & -1.302957 \\
7 & 6 & 0 & 2.539044 & 1.288514 & -1.305426 \\
8 & 7 & 0 & 3.374607 & 0.159329 & -1.270166 \\
9 & 6 & 0 & 2.649645 & -1.039189 & -1.299544 \\
10 & 6 & 0 & -2.539110 & -1.290148 & -1.303904 \\
11 & 7 & 0 & -3.374675 & -0.160921 & -1.270067 \\
12 & 6 & 0 & -2.649713 & 1.037564 & -1.300870 \\
& & & & S19 &
\end{tabular}




\begin{tabular}{|c|c|c|c|c|c|}
\hline 13 & 8 & 0 & 2.897363 & 2.445820 & -1.358385 \\
\hline 14 & 8 & 0 & 3.126253 & -2.155417 & -1.339984 \\
\hline 15 & 8 & 0 & -2.897409 & -2.447526 & -1.355404 \\
\hline 16 & 8 & 0 & -3.126331 & 2.153732 & -1.342804 \\
\hline 17 & 6 & 0 & -4.839975 & -0.215027 & -1.241235 \\
\hline 18 & 6 & 0 & -5.442059 & 0.264581 & 0.063425 \\
\hline 19 & 6 & 0 & 4.839909 & 0.213519 & -1.241509 \\
\hline 20 & 6 & 0 & 5.442100 & -0.264454 & 0.063695 \\
\hline 21 & 6 & 0 & -6.064002 & 1.508873 & 0.155138 \\
\hline 22 & 6 & 0 & -6.643434 & 1.945497 & 1.349397 \\
\hline 23 & 6 & 0 & -6.594635 & 1.122165 & 2.470922 \\
\hline 24 & 6 & 0 & -5.972432 & -0.128144 & 2.410171 \\
\hline 25 & 6 & 0 & -5.394553 & -0.555291 & 1.210564 \\
\hline 26 & 6 & 0 & 5.394604 & 0.556806 & 1.209837 \\
\hline 27 & 6 & 0 & 5.972573 & 0.131141 & 2.409926 \\
\hline 28 & 6 & 0 & 6.594852 & -1.119057 & 2.472164 \\
\hline 29 & 6 & 0 & 6.643645 & -1.943747 & 1.351634 \\
\hline 30 & 6 & 0 & 6.064121 & -1.508599 & 0.156881 \\
\hline 31 & 8 & 0 & 4.759063 & 1.752123 & 1.043040 \\
\hline 32 & 6 & 0 & 4.697796 & 2.657932 & 2.133193 \\
\hline 33 & 8 & 0 & -4.759089 & -1.750850 & 1.045180 \\
\hline 34 & 6 & 0 & -4.697910 & -2.655407 & 2.136374 \\
\hline 35 & 1 & 0 & 0.120082 & -2.530639 & -1.308063 \\
\hline 36 & 1 & 0 & -0.120149 & 2.529005 & -1.311315 \\
\hline 37 & 1 & 0 & -5.095540 & -1.259553 & -1.433374 \\
\hline 38 & 1 & 0 & -5.213785 & 0.399782 & -2.065154 \\
\hline 39 & 1 & 0 & 5.095388 & 1.257830 & -1.434916 \\
\hline 40 & 1 & 0 & 5.213708 & -0.402258 & -2.064713 \\
\hline 41 & 1 & 0 & -6.081415 & 2.148072 & -0.723223 \\
\hline 42 & 1 & 0 & -7.122483 & 2.918636 & 1.399424 \\
\hline 43 & 1 & 0 & -7.040417 & 1.446313 & 3.407670 \\
\hline 44 & 1 & 0 & -5.942833 & -0.756931 & 3.292720 \\
\hline 45 & 1 & 0 & 5.942973 & 0.760980 & 3.291725 \\
\hline 46 & 1 & 0 & 7.040701 & -1.442035 & 3.409283 \\
\hline 47 & 1 & 0 & 7.122751 & -2.916797 & 1.402814 \\
\hline 48 & 1 & 0 & 6.081520 & -2.148862 & -0.720705 \\
\hline 49 & 1 & 0 & 4.148237 & 2.229830 & 2.981864 \\
\hline 50 & 1 & 0 & 4.163846 & 3.532419 & 1.758391 \\
\hline 51 & 1 & 0 & 5.700909 & 2.955043 & 2.465778 \\
\hline 52 & 1 & 0 & -5.701052 & -2.952014 & 2.469320 \\
\hline 53 & 1 & 0 & -4.148286 & -2.226393 & 2.984544 \\
\hline 54 & 1 & 0 & -4.164069 & -3.530387 & 1.762570 \\
\hline
\end{tabular}

\section{References}

(1) Okada, T.; Fujiwara, N.; Ogata, T.; Haba, O.; Ueda, M. J. Polym. Sci., Part A: Polym. Chem. 1997, 35, 2259-2266.

(2) Sasaki, S.; Mizuno, M.; Naemura, K.; Tobe, Y. J. Org. Chem. 2000, 65, 275-283. 